\title{
BMJ Open Development and pilot testing of a decision aid for the initiation of antipsychotic medications in persons with dementia in long-term care using a systematic approach: a study protocol
}

\author{
Leslie J Malloy-Weir, ${ }^{1}$ Andrew Kirk ${ }^{2}$
}

To cite: Malloy-Weir LJ, Kirk A. Development and pilot testing of a decision aid for the initiation of antipsychotic medications in persons with dementia in long-term care using a systematic approach: a study protocol. BMJ Open 2017;7:e018769. doi:10.1136/ bmjopen-2017-018769

- Prepublication history for this paper is available online. To view these files, please visit the journal online (http://dx.doi org/10.1136/bmjopen-2017018769).

Received 21 July 2017 Revised 14 September 2017 Accepted 22 September 2017

CrossMark

${ }^{1}$ Department of Psychology, University of Saskatchewan, Saskatoon, Canada

${ }^{2}$ College of Medicine, University of Saskatchewan, Saskatoon, Canada

Correspondence to Dr Leslie J Malloy-Weir; Imalloyw@gmail.com

\section{ABSTRACT}

Introduction Antipsychotic medications are commonly used in long-term care to treat neuropsychiatric symptoms of dementia despite concerns that their risks (eg, infection, falls, death) may outweigh their benefits. This study protocol outlines the development and pilot testing of a decision aid for antipsychotic medications that is tailored to the information needs of residents with dementia in long-term care and family caregivers (or decision makers). The goals of the decision aid are to help residents and caregivers (1) better understand the risks and benefits of antipsychotic medications in long-term care, and (2) make informed decisions about their use (or non-use).

Methods and analysis This multiphased study is being conducted between October 2016 and September 2018. In phase I, the decision aid will be developed after consultation with a steering group, review of scientific evidence on outcomes associated with pharmacological treatments for neuropsychiatric symptoms of dementia in long-term care, review of guidelines for the use of antipsychotic medications in long-term care, and review of guidelines for writing health information for patients and families. The decision aid will also be alpha-tested and redrafted, as necessary, in phase I. In phase II, implementation and reporting guidelines for the decision aid will be developed in collaboration with Directors of Care in long-term care. In phase III, the decision aid will be (1) beta-tested with residents with dementia in long-term care and caregivers not involved in the design phase, and (2) assessed by an external panel of experts.

Ethics and dissemination Ethical approval for this study has been granted by the Research Ethics Board at the University of Saskatchewan, approval number Beh 16-465. Findings from this study will be disseminated via conference presentations, publications, presentations to policy makers and plain language summaries to residents with dementia in long-term care and their caregivers.

\section{INTRODUCTION}

Dementia is 'typically defined as a clinical syndrome of cognitive decline that is sufficiently severe to interfere with social or occupational functioning' (p2). ${ }^{1}$ In 2015, the
Strengths and limitations of this study

- A systematic and participatory approach will increase the acceptability of the decision aid and better prepare residents with dementia in long-term care and their family caregivers (or decision makers) to make an informed decision about the use (or nonuse) of antipsychotic medications.

- The development of implementation and reporting guidelines, in collaboration with Directors or Care, will ensure that the decision aid is implemented in a respectful, safe and ethical manner in the long-term care setting.

- The non-random design used in the beta testing phase of this study will not allow causal inferences to be drawn from the findings. The findings will, however, be useful for designing and conducting a randomised controlled trial and economic evaluation of the decision aid.

prevalence of dementia worldwide was estimated at 47 million. This number is expected to increase to 75 million in $2030 .^{2}$

The progressive nature of Alzheimer's disease and related dementias presents challenges to both the independence and care of those directly affected, particularly in the middle and late stages. As such, persons with dementia comprise a significant proportion of adults living in care homes, with estimates ranging from $30 \%$ to $50 \%$ in high-income countries. ${ }^{3}$ Neuropsychiatric symptoms (eg, delusions, hallucinations, aggression) are common in people diagnosed with Alzheimer's disease. ${ }^{4}$ In long-term care settings, antipsychotic medications are commonly used to treat neuropsychiatric symptoms of dementia (eg, physical aggression that may harm oneself or others) despite concerns that the risks associated with these medications (eg, infection, falls, death) may outweigh their benefits. ${ }^{56}$ Guidelines for antipsychotic drug 
use for dementia in care homes thus recommend, among other things, that the "Family caregiver should be informed and consulted throughout treatment and discontinuation' (p1854). ${ }^{7}$ Missing from these guidelines, however, is guidance on (1) if, and how, the risks and benefits of antipsychotic medications should be communicated to the patient (if possible) and family caregiver (or decision maker), or (2) the extent to which the patient and family caregiver (or decision maker) should be involved in decisions about the use of these medications.

\section{Treatment decision-making framework}

In Charles $e t a l$ s treatment decision-making framework, ${ }^{8}$ the treatment decision-making process is described as dynamic and can unfold in different ways depending on the one or more model(s) adopted (eg, paternalistic, shared, informed). In the paternalistic model, the minimum amount of legally required information is communicated by the physician to the patient. The physician deliberates on the treatment decision alone or with other physicians. The decision on the treatment to implement is also made by the physician(s). In the shared model, all relevant information (ie, medical and personal) is communicated between the physician and patient. The physician, patient and potentially others deliberate on the treatment decision together. The decision on the treatment to implement is made by both the physician and the patient. Fundamental to the shared model is a perception among physicians and patients that treatment choices exist. The informed model differs from the other models in that all relevant information for decision making is communicated primarily from the physician to the patient. The patient and potentially others deliberate on the treatment decision, while the decision on the treatment to implement is made by the patient alone. In between approaches are situated between the three predominant models (ie, paternalistic, shared, informed) and, as the name implies, possess characteristics of the two bordering models.

\section{Patient decision aids}

Internationally, there is an increasing trend towards the implementation of shared decision making and the use of patient decision aids. ${ }^{9}$ Patient decision aids are tools that 'provide information on the options and help patients clarify and communicate the personal value they associate with different features of the options' (10 para 1). They 'do not advise people to choose one option over another, nor are they meant to replace practitioner consultation. Instead, they prepare patients to make informed, valuesbased decisions with their practitioner' (para 2). ${ }^{10}$

A Cochrane review has found the use of decision aids to be associated with an increased knowledge of treatment options and lower decisional conflict in patients. ${ }^{11}$ Few studies ${ }^{12}{ }^{13}$ have examined the use of decision aids in dementia-related care despite evidence that persons with dementia (1) wish to be involved in the decision-making process, (2) are capable of communicating their values at both the moderate and severe stages of the disease, and (3) may be prematurely excluded from decisions about their care. ${ }^{14}$ Given the widespread and persistent use of antipsychotic medications for neuropsychiatric symptoms of dementia in long-term care, the risks associated with their use and an increasing trend towards the use of decision aids for making complex decisions, the primary aim of this study is to develop and pilot-test a decision aid for antipsychotic medications in long-term care that is tailored to the information needs of residents with dementia and their family caregiver(s) (or decision makers). A secondary aim of this study is to evaluate the feasibility of our methods for use in a larger randomised controlled trial. The goals of the decision aid are to help residents with dementia in long-term care and their family caregivers (or decision makers) (1) better understand the risks and benefits associated with antipsychotic medications in long-term care, and (2) make an informed decision about their use (or non-use).

\section{METHODS AND ANALYSIS}

To meet the aims of this study, a systematic development process for decision aids has been adopted. ${ }^{15}$ Key features of this process are summarised in figure 1 and described in the following sections.

\section{Phase 1}

Scope, purpose and target audience

The health condition, or problem, is neuropsychiatric symptoms (eg, delusions, hallucinations, aggression) exhibited by residents with dementia in long-term care. The treatment decision that needs to be considered is the use (or non-use) of antipsychotic drugs. The target audience is residents with dementia in long-term care exhibiting neuropsychiatric symptoms and/or their family caregivers (or decision makers).

The Government of Canada defines long-term care as facilities that 'provide living accommodation for people who require on-site delivery of 24 hours, 7 days a week supervised care, including professional health services, personal care and services such as meals, laundry and housekeeping' (para 1). ${ }^{16}$ Long-term care facilities located within a single health region in Saskatchewan, Canada, will provide the setting for this study.

\section{Steering group}

A steering group, which is comprised residents with dementia in long-term care (if possible), family caregivers of, or decision makers for, residents with dementia in long-term care, and health professionals caring for residents with dementia in long-term care (eg, nurses, psychiatrist, psychologist, pharmacist), will be formed. Members of the steering group $(n=8-10)$ will be recruited with the help of a manager who works within our designated health region and who possesses expertise in the care of seniors. To facilitate the recruitment process, an email invitation containing information about the study will be 


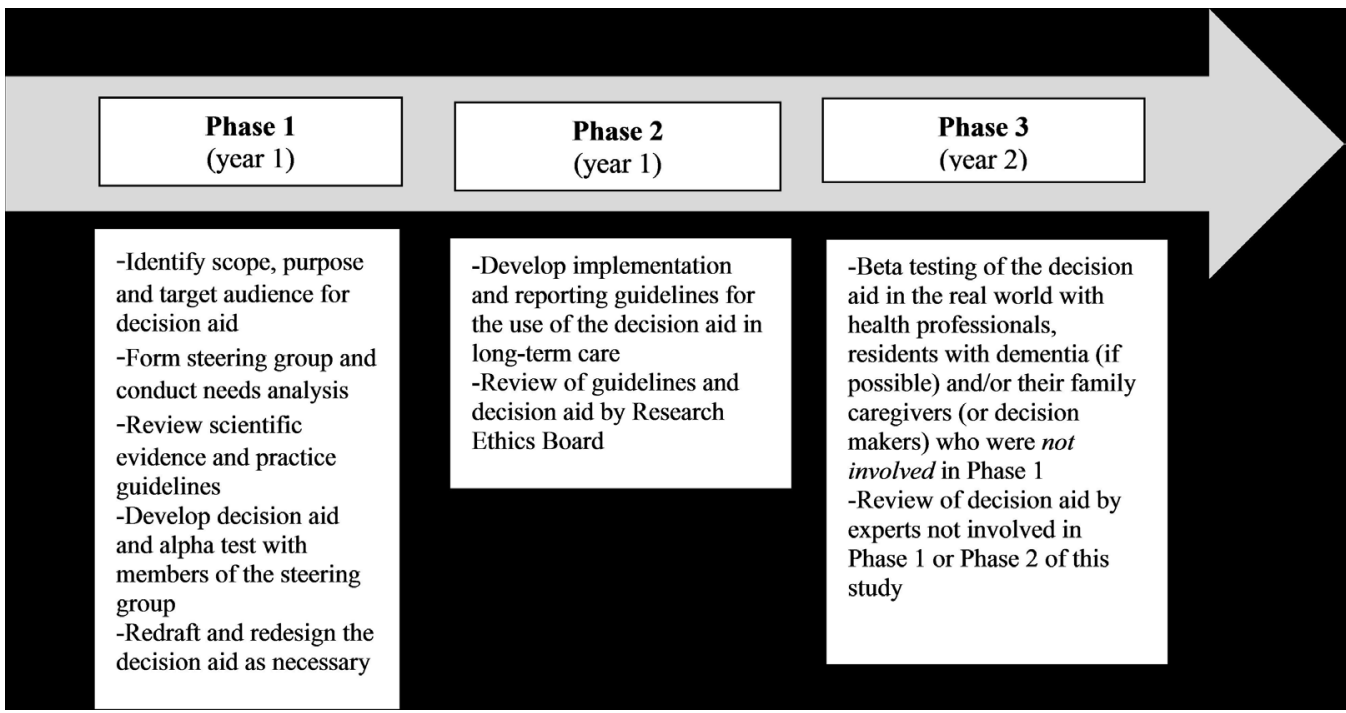

Figure 1 Timeline for development of decision aid.

sent to health professionals working in long-term care in our health region. Information about the study will also be provided to residents with dementia in long-term care and their family caregivers (or decision makers). Verbal or written consent will be obtained from health professionals and family caregivers (or decision makers) prior to their participation in this study. An assent process will be used with residents with dementia in long-term care who agree to participate. Since members of the steering group will be consulted at multiple points in time throughout this study, the consent or assent process will be repeated prior to each consultation.

\section{Content and design of decision aid}

The content of the decision aid will be guided by a needs analysis, scientific evidence, guidelines for antipsychotic drug use for dementia in long-term care and the Ottawa patient decision aid template. ${ }^{17}$ The Ottawa template is divided into multiple sections that allow developers to insert information on who and what the decision aid is for, the available treatment option(s), the benefits, risks and side effects associated with the treatment option(s), and other information that may affect treatment decision making (eg, personal stories). A series of steps are also included in the template to help the target audience compare options, identify what matters most, what else is needed to prepare for decision making and the next steps.

To determine the information needs of residents with dementia and/or the family caregivers (or decision makers), semistructured interviews will be conducted with members of the steering group who voluntarily agree to participate and who consent or assent. Interviewees will be asked, among other things, to identify the type(s) of information needed to make an informed decision about antipsychotic medications as well as the preferred format(s) for this information. Interviews will be conducted one-on-one, in person or by phone to ensure confidentiality and avoid bias from other steering group members. Interviews will be digitally recorded with the permission of participants and will be transcribed verbatim. Identifying information will be removed from any summaries, reports or presentations of the findings to protect the anonymity of participants. Interview data will be thematically analysed ${ }^{18}$ by at least two members of the research team. This analysis will involve repeated review of interview transcripts to become familiar with the data, the generation of initial codes, a search for themes, a review of themes, the creation of a thematic map, and the generation of names and clear definitions for each theme. A plain language summary of the findings will be reviewed, confirmed and/or modified by members of the steering group. A scholarly report of the analysis will be written for publication purposes.

Scientific evidence on outcomes associated with the use of antipsychotic drugs on neuropsychiatric symptoms in long-term care and the limitations of this evidence will also be identified, reviewed and summarised. This will involve the updating of a previously conducted systematic review of pharmacological treatments for neuropsychiatric symptoms of dementia in long-term care. ${ }^{6}$ A separate review will be conducted of (1) international, national, provincial, regional and institutional level guidelines for antipsychotic drug use for dementia in long-term care, and (2) adverse events reported, or government warnings issued, for antipsychotic drugs.

The design of the decision aid will be guided by the steering group's views on preferred information format(s) (eg, paper-based booklet, video, internet), as well as guidelines for writing health information for patients and families. ${ }^{19}$ This will ensure that information contained in the decision aid is logically ordered as well as easily understood by, and acceptable to, the target audience. 


\section{Alpha testing}

Members of the steering group who voluntarily agree to participate in the alpha testing phase will be asked to assess the comprehensibility and usability of the decision aid using a 10-item survey adapted from the Ottawa acceptability tool. ${ }^{20}$ This survey assesses respondents' perceptions about the information received (eg, length, amount, usefulness for decision making, suggestions for improvement) using a combination of closed-ended and open-ended response options. Responses to quantitative survey questions will be summarised using descriptive statistics. Qualitative responses will undergo thematic analysis ${ }^{18}$ and will be used to interpret quantitative findings. Overall findings from the survey will be used to redesign and redraft the decision aid, as necessary.

Assessments of the decision aid's comprehensibility and usability will be repeated until it is deemed ready for use in our long-term care setting. In order to be deemed ready for use, the decision aid must achieve a high level of acceptability among members of the steering group. The decision aid must also meet relevant patient decision aid certification criteria established by the Washington State Health Care Authority. ${ }^{21}$ Relevant criteria include, among other things, whether or not the decision aid contains a description of the health condition or problem, explicitly states the decision under consideration, identifies the target audience, describes the positive and negative features of each option, follows plain language guidelines, discloses funding sources, and includes the authors/developers' credentials or qualifications. Any disagreements that arise over the content and design of the decision aid, as well as steps taken to resolve them, will be recorded and reported as part of our study findings. Recommended and actual changes made to the decision aid over the course of its development and testing will also be documented and reported.

\section{Phase 2}

\section{Implementation and reporting guidelines}

In the province of Saskatchewan, Canada, long-term care homes are 'owned and operated by municipalities, religiously affiliated organizations, and, private, for-profit organizations' (p1).22 Directors of Care, typically registered nurses by training, develop and oversee policy in these homes and perform, among other things, leadership and administrative tasks (eg, education, training, budget). In phase II, semistructured interviews will be conducted with the Director of Care to ascertain (1) how the decision aid will be administered in practice, (2) how the results from the decision aid will be shared, (3) if the use of the decision aid will be entered into the medical record, (4) steps to be taken if a disagreement arises between the resident/family caregiver and physician over the use of antipsychotic medications, and (5) how any unintended consequences associated with the administration of the decision aid in long-term care will be reported and handled. After their development, the guidelines, along with the decision aid, will be reviewed by the
Research Ethics Board at the University of Saskatchewan. This will ensure that the decision aid will be implemented in a respectful, safe and ethical manner during the beta testing phase.

\section{Phase 3}

\section{Beta testing}

In the beta testing phase, the feasibility of the decision aid will be evaluated under real-life conditions and by individuals $(\mathrm{n}=18-20)$ who were not involved in the design phase. The long-term care facility(ies) in which beta testing will occur will be selected with the assistance of our regional partner (ie, manager who works within our designated health region and who possesses expertise in the care of seniors) and after consultation with the Director(s) of Care and any staff involved in the administration and testing of the decision aid in that setting.

Pre-exposure and post-exposure to the decision aid, residents with dementia in long-term care and/or their family caregivers (or decision makers) will be assessed in terms of their (1) knowledge about the benefits and risks of antipsychotic medications using an 8-10-item survey based on the Ottawa Knowledge Questionnaire template ${ }^{23}$; (2) treatment preference; (3) level of decisional conflict using the low-literacy decisional conflict scale $^{24}$; and (4) satisfaction with the decision using the satisfaction with decision scale. ${ }^{25} \mathrm{~A}$ paired t-test will be used to compare pre-exposure and post-exposure knowledge, decisional conflict and satisfaction with decision. Changes in treatment preference pre-exposure and postexposure to the decision aid will be analysed using McNemar's test. A sample size of $n=16$ is required to achieve $80 \%$ power with type I error $\alpha=0.05$, estimated effect size $=0.35$ and correlation among repeated measures $=0.6 .^{26} 27$

Interviews will be conducted with health professionals, residents with dementia in long-term care and family caregivers (or decision makers) exposed to the decision aid to obtain their answers to the following questions: Was the decision aid useful in helping you to make a treatment decision? What did you like about the decision aid? How do you think the decision aid could be improved ? $^{28}$ Unintended consequences (ie, positive and negative) related to the use of the decision aid and the accompanying implementation and reporting guidelines will also be explored with interview participants in phase III. Qualitative data will be thematically analysed ${ }^{18}$ and will be used to interpret quantitative findings.

The final step in phase III involves the formation of a panel of experts $(n=3-5)$, external to this study, who will assess the decision aid in terms of its content and design. Members of this panel will be identified through a review of the scientific literature on patient decision aids. The panel will be provided information about the long-term care setting as well as a copy of the implementation and reporting guidelines for the decision aid in that setting. Panel members' feedback on the decision aid will be reported as part of the study findings and will be used 
to further refine the decision aid, and the guidelines regarding its use, if necessary.

\section{Timeline}

This study will be conducted over a 2-year period (October 2016 to September 2018). Development and alpha testing of the decision aid will take place in year 1. Beta testing of the decision aid will occur in year 2. Updating of the 2013 systematic review on pharmacological treatments for neuropsychiatric symptoms of dementia in long-term care is at its near completion. One-on-one interviews with members of the steering group to identify the type(s) of information needed to make an informed decision about antipsychotic medications as well as the preferred format(s) to receive or provide this information are also at its near completion.

\section{ETHICS AND DISSEMINATION}

The methods described in this study protocol have received approval from the Research Ethics Board at the University of Saskatchewan, approval number Beh 16-465. Data will be collected, analysed, reported and stored in accordance with the conditions set out by the Research Ethics Board at the University of Saskatchewan.

The findings of this study will be widely disseminated via presentations at relevant academic conferences, publications in peer-reviewed scientific journals, plain language summaries to residents with dementia and family caregivers (or decision makers), and presentations to policy makers at the institutional, regional and provincial levels. Issues that arise during all phases of the study will be recorded and reported. Disagreements over the content, design and implementation of the decision aid, and how these disagreements were handled, will also be recorded and reported.

Findings from this study will be of interest to researchers and policy makers in Canada and internationally given the projected increase in the prevalence of dementia worldwide, the widespread and persistent use of antipsychotic medications in long-term care for neuropsychiatric symptoms of dementia, and an increasing trend towards the use of patient decision aids. Findings from this study will be used to design and conduct a randomised controlled trial, as well as an economic evaluation of the decision aid.

Acknowledgements We are grateful to Karen Lawson (Department of Psychology, University of Saskatchewan) for critically reviewing the protocol and contributing to the revisions of the manuscript. We are also grateful to Dawn Stacey (School of Nursing, University of Ottawa) for recommending the use of the Washing State Health Care Authority patient decision aid certification criteria in our study.

Contributors LJM-W designed the study and prepared the manuscript. AK contributed to the conceptual development of the study and to the revisions of the manuscript.

Funding This work is supported by a Postdoctoral Fellowship awarded to LJM-W by the Saskatchewan Health Research Foundation.

Competing interests None declared.
Ethics approval Ethical approval for this study has been granted by the Research Ethics Board at the University of Saskatchewan, approval number Beh 16-465.

Provenance and peer review Not commissioned; externally peer reviewed.

Open Access This is an Open Access article distributed in accordance with the Creative Commons Attribution Non Commercial (CC BY-NC 4.0) license, which permits others to distribute, remix, adapt, build upon this work non-commercially, and license their derivative works on different terms, provided the original work is properly cited and the use is non-commercial. See: http://creativecommons.org/ licenses/by-nc/4.0/

(C) Article author(s) (or their employer(s) unless otherwise stated in the text of the article) 2017. All rights reserved. No commercial use is permitted unless otherwise expressly granted.

\section{REFERENCES}

1. Chertkow $\mathrm{H}$, Feldman $\mathrm{HH}$, Jacova $\mathrm{C}$, et al. Definitions of dementia and predementia states in Alzheimer's disease and vascular cognitive impairment: consensus from the Canadian conference on diagnosis of dementia. Alzheimers Res Ther 2013;5(Suppl 1):S2.

2. Alzheimer's Disease International. World Alzheimer Report 2015. www.alz.co.uk/research/worldalzheimerreport2015summary.pdf. (accessed 5 Jul 2017).

3. Alzheimer's Disease International. World Alzheimer Report 2013. www.alz.co.uk/research/world-report-2013. (accessed 5 Jul 2017).

4. Lyketsos CG, Carrillo MC, Ryan JM, et al. Neuropsychiatric symptoms in Alzheimer's disease. Alzheimers Dement 2011;7:532-9.

5. Canadian Institute for Health Information. Use of Antipsychotics among seniors living in long-term care facilities. $2016 \mathrm{https} / / /$ secure. cihi.ca/estore/productFamily.htm?locale=en\&pf=PFC3094 (accessed 5 Jul 2017).

6. Seitz DP, Gill SS, Herrmann N, et al. Pharmacological treatments for neuropsychiatric symptoms of dementia in long-term care: a systematic review. Int Psychogeriatr 2013;25:185-203.

7. Zuidema SU, Johansson A, Selbaek G, et al. A consensus guideline for antipsychotic drug use for dementia in care homes. Bridging the gap between scientific evidence and clinical practice. Int Psychogeriatr 2015;27:1849-59.

8. Charles C, Gafni A, Whelan T. Decision-making in the physicianpatient encounter: revisiting the shared treatment decision-making model. Soc Sci Med 1999;49:651-61.

9. Härter M, Moumjid N, Cornuz J, et al. Shared decision making in 2017: international accomplishments in policy, research and implementation. Z Evid Fortbild Qual Gesundhwes 2017;123124:1-5.

10. International Patient Decision Aid Standards (IPDAS) Collaboration. What are patient decision aids? 2012 http://ipdas.ohri.ca/what.html (accessed 5 Jul 2017).

11. Stacey D, Legare F, Lewis K, et al. Decision aids for people facing health treatment or screening decisions(Review). The Cochrane Library 2017 https://decisionaid.ohri.ca/docs/develop/ Cochrane_ Review.pdf (accessed 5 Jul 2017).

12. Volandes AE, Ferguson LA, Davis AD, et al. Assessing end-oflife preferences for advanced dementia in rural patients using an educational video: a randomized controlled trial. J Palliat Med 2011;14:169-77.

13. Volandes AE, Paasche-Orlow MK, Barry MJ, et al. Video decision support tool for advance care planning in dementia: randomised controlled trial. BMJ 2009;338:b2159.

14. Miller LM, Whitlatch CJ, Lyons KS. Shared decision-making in dementia: A review of patient and family carer involvement. Dementia 2016;15:1-17.

15. Coulter A, Stilwell D, Kryworuchko J, et al. A systematic development process for patient decision aids. BMC Med Inform Decis Mak 2013;13(Suppl 2):S2.

16. Government of Canada. What is long-term facilities-based care? 2004 https://www.canada.ca/en/health-canada/services/homecontinuing-care/long-term-facilities-based-care.html (accessed $5 \mathrm{Jul}$ 2017).

17. Ottawa Hospital Research Institute. Development method for Ottawa patient decision aids. $2015 \mathrm{https}$ ://decisionaid.ohri.ca/methods.html (accessed 5 Jul 2017).

18. Braun V, Clarke V. Using thematic analysis in psychology. Qual Res Psychol 2006;3:77-101.

19. Wizowski L, Harper T, Hutchings T. Writing health information for patients and families. $2014 \mathrm{http}: / /$ www.hamiltonhealthsciences. ca/workfiles/PATIENT_ED/Writing_HI_Edition4.pdf (accessed 5 Jul 2017). 
20. Ottawa Hospital Research Institute. Patient decision aids. Acceptability. 2015 https://decisionaid.ohri.ca/docs/develop/Tools/ Acceptability_osteoporosis.pdf (accessed 5 Jul 2017).

21. Washington State Health Care Authority. Patient decision aids. 2017 https://www.hca.wa.gov/about-hca/healthier-washington/patientdecision-aids-pdas (accessed 5 Jul 2017).

22. Sykes Assistance Services Corporation. Long term care in Saskatchewan 2016. 2016 https://www.sunnet.sunlife.com/files/ advisor/english/PDF/Completereport-LTC-Costs-SK.pdf (accessed 30 Aug 2017).

23. Ottawa Hospital Research Institute. Patient decision aids. Knowledge 2014 https://decisionaid.ohri.ca/docs/develop/Tools/Knowledge_ Tamoxifen.pdf (accessed 5 Jul 2017).
24. O'Connor AM.Low literacy decisional conflict scale. $2010 \mathrm{https} / / /$ decisionaid.ohri.ca/docs/develop/Tools/DCS_LowLiteracy_English. pdf (accessed 5 Jul 2017).

25. Holmes-Rovner M, Kroll J, Schmitt N, et al. Patient satisfaction with health care decisions: the satisfaction with decision scale. Med Decis Making 1996;16:58-64.

26. Faul F, Erdfelder E, Lang AG, et al. G*Power 3: a flexible statistical power analysis program for the social, behavioral, and biomedical sciences. Behav Res Methods 2007;39:175-91.

27. Cohen J. Statistical power analysis for the behavioral sciences. 2nd ed. Hillsdale: NJLawrence Earlbaum Associates, 1988.

28. Carmody J, Potter J, Lewis K, et al. Development and pilot testing of a decision aid for drivers with dementia. BMC Med Inform Decis Mak 2014;14:19. 